\title{
Spatially distributed control for optimal drag reduction of the flow past a circular cylinder
}

\author{
By PHILIPPE PONCET ${ }^{1,2}$, ROLAND HILDEBRAND ${ }^{3}$, \\ GEORGES-HENRI COTTET ${ }^{3}$ AND PETROS KOUMOUTSAKOS \\ ${ }^{1}$ Université de Toulouse, INSA, GMM 135 avenue de Rangueil, F-31077 Toulouse, France \\ ${ }^{2}$ CNRS, Institut de Mathématiques de Toulouse, Equipe MIP, F-31077 Toulouse, France \\ ${ }^{3}$ Laboratoire Jean Kuntzmann, CNRS and Université de Grenoble, BP 53, F-38041 Grenoble, France \\ ${ }^{4}$ Computational Science, ETH Zurich, CH-8092, Switzerland
}

(Received November 9th 2007)

\begin{abstract}
We report high drag reduction in Direct Numerical Simulations of controled flows past circular cylinders at Reynolds numbers of 300 and 1000. The flow is controlled by the azimuthal component of the tangential velocity of the cylinder surface. Starting from a spanwise uniform velocity profile that leads to high drag reduction, the optimization procedure identifies, for the same energy input, spanwise varying velocity profiles that lead to higher drag reduction. The three dimensional variations of the velocity field, corresponding to the modes A and B of three dimensional wake instabilities, are largely responsible for this drag reduction. The spanwise, wall velocity variations introduce streamwise vortex braids in the wake that are responsible for reducing the drag induced by the primary spanwise vortices shed by the cylinder. The results demonstrate that extending two dimensional controllers to three-dimensional flows is not optimal as three dimensional control strategies can lead efficiently to higher drag reduction.
\end{abstract}

\section{Introduction}

What is the maximum drag reduction that can be achieved in bluff body flows for a given energy of the controller? Are two-dimensional control strategies sufficient for drag reduction in three-dimensional flows? We address these questions via Direct Numerical Simulations (DNS) of flows past circular cylinders controlled by spatial variations of the azimuthal component of the wall velocity.

Circular cylinders are widely considered as prototypical configurations for the study of bluff body flows. Drag inducing mechanisms of cylinder flows involve a nonlinear interaction between the vortical structures in the wake and the generation of vorticity on the cylinder surface. The three dimensional instabilities of the wake (Barkley \& Henderson (1996) Williamson (1996b)) are related to mechanisms of drag production, while modifications of the cylinder shape and its velocity can be optimized in order to reduce the drag of the flow (Choi et al. (2008)).

In this work we are interested in drag reduction induced by modifications of the tangential component of the wall velocity. A uniform, albeit unsteady, velocity on the cylinder surface was shown to induce very high drag reduction in experiments (Tokumaru \& Dimotakis (1991)) and simulations (Dennis et al. (2000), Poncet (2004)) of cylinders undergoing rotary oscillations. Additional controllers can be devised by modifying the wall velocity profile (Kim \& Choi (2005), Milano \& Koumoutsakos (2002)). In Milano \& Koumoutsakos (2002) two dimensional simulations were performed for flows past a circular cylinder controlled by azimuthal variations 
of the tangential wall velocity. The optimal velocity profile was obtained using an evolutionary optimization procedure leading to high drag reduction. This velocity profile was then extended uniformly in the spanwise direction leading to drag reduction for three dimensional flows (Poncet et al. (2005), Poncet (2005)). Hence one may conjecture that two-dimensional control strategies obtained from relevant experiments and simulations would provide an efficient method of identifying optimal wall velocity profiles that can then be extended to the control of threedimensional flows. Open issues include the robustness of the velocity profiles with respect to three-dimensional perturbations as well as the extent to which the efficiency of two-dimensional control strategies could be improved using three-dimensional wall velocity profiles. There is mounting evidence (Lim \& Lee (2004), Kim \& Choi (2005), Dobre et al. (2006)) that threedimensional controllers may indeed lead to enhanced drag reduction that is beyond what is possible by two-dimensional controllers. This evidence complements the fact that, in uncontrolled flows, three dimensional instabilities in the cylinder wake are associated with drag levels which are below those of the base two-dimensional flows.

In this paper we consider a systematic development of three-dimensional control strategies for cylinder flows by varying the distributions of azimuthal component of the velocity on the cylinder surface. We start from an optimal, spanwise uniform profile that has been obtained for 2D cylinder wakes in Milano \& Koumoutsakos (2002). We then consider, for a given control energy, parametric modulations of this profile along the spanwise direction. We identify, using a simple design of experiments approach (Box \& Wilson (1951)), optimal parameters that result in spanwise velocity variations leading to drag reduction that is over four times more efficient than the initial 2D control strategy. We note that flow control using spanwise variations of the wall velocity has been presented before in Kim \& Choi (2005). The control parameters in the present work differ from those in Kim \& Choi (2005) in two respects. First, we consider tangential velocities instead of blowing-suction. Second, in Kim \& Choi (2005) large wavelengths are considered for the modulation of the cylinder. In the present work we use harmonic variations, instead of sub-harmonics, of the cylinder span as for a given energy level this choice produces higher levels of axial vorticity. The production of axial vorticity near the cylinder surface plays an important role in drag reduction as it is further discussed in the next section.

The paper is structured as follows: In section 2 we outline the computational methods used in the present study and the evaluation of drag coefficient. In section 3 we present the results of the optimization strategy for 3D flows and we conclude in section 4 with a discussion of the flow characteristics as they are related to drag reduction mechanisms.

\section{Incompressible flows past circular cylinders}

We consider the three-dimensional flow of an incompressible viscous fluid past a circular cylinder at Reynolds numbers $\left(R e=U_{\infty} D / \nu\right)$ of 300 and 1000 , where $U_{\infty}, \nu$ denote respectively the free stream velocity and the viscosity of the fluid and $D=2 R$ denotes the diameter of the cylinder. A representative view of the cylinder wake structures as obtained in experiments Williamson (1996b) and in DNS Poncet (2004) is depicted in Figure 2.

\subsection{Governing Equations and Numerical Methods}

We consider the incompressible Navier-Stokes equations in their vorticity-velocity $(\boldsymbol{\omega}-\boldsymbol{u})$ formulation :

$$
\frac{\partial \boldsymbol{\omega}}{\partial t}+\boldsymbol{u} \cdot \boldsymbol{\nabla} \boldsymbol{\omega}=\boldsymbol{\omega} \cdot \boldsymbol{\nabla} \boldsymbol{u}+\nu \Delta \boldsymbol{\omega}
$$

with $\boldsymbol{\omega}=\nabla \times \boldsymbol{u}$ and $\nabla \cdot \boldsymbol{u}=0$. These equations are complemented with the no-slip boundary condition on the moving surface of the cylinder. Using a cylindrical coordinate system 


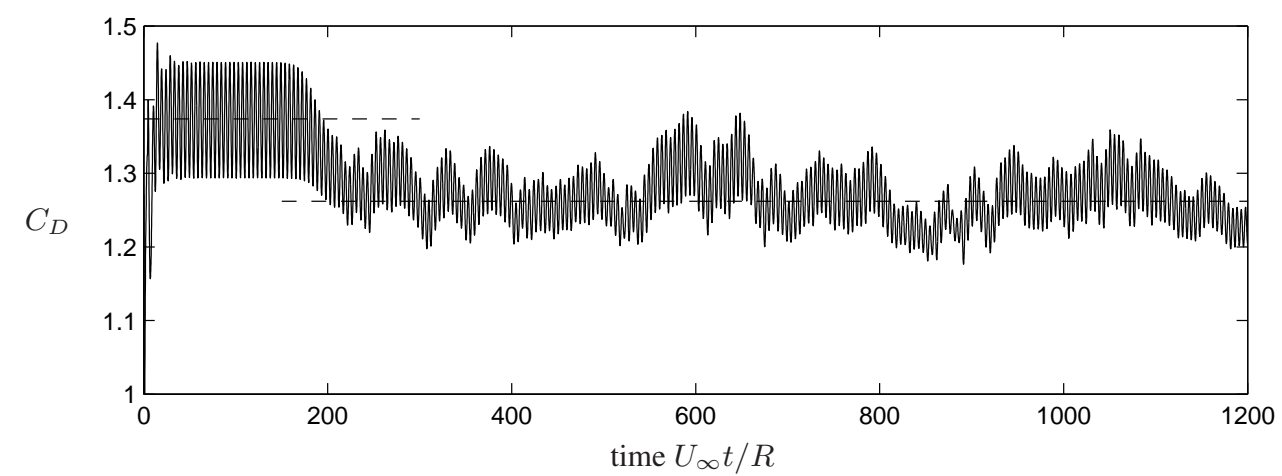

FIGURE 1. Evolution of the drag coefficient for a three-dimensional cylinder wake at $R e=300$ (the dashed lines denote the reference values 1.374 and 1.262). The drag coefficient is initially periodic corresponding to a $2 \mathrm{D}$ flow, until 3D instabilities become the dominant feature, leading to an non-periodic signal and a lower drag value.

$\left(\boldsymbol{e}_{r}, \boldsymbol{e}_{\theta}, \boldsymbol{e}_{z}\right)$ these boundary conditions are expressed as

$$
\boldsymbol{u}_{r=R}=U_{r}(\theta, z, t) \boldsymbol{e}_{r}+U_{\theta}(\theta, z, t) \boldsymbol{e}_{\theta}+U_{z}(\theta, z, t) \boldsymbol{e}_{z}
$$

In the present simulations the spanwise and normal wall velocity components are set to zero. The flow is controlled by varying the steady azimuthal component of the wall velocity $\left(U_{\theta}(\theta, z)\right)$. Throughout this paper we consider a cylinder of radius 1 . The computational box is the cylindrical domain $1 \leqslant r \leqslant R_{\max }, 0 \leqslant \theta \leqslant 2 \pi,-L / 2 \leqslant z \leqslant L / 2$, and on the outer boundary ( $r=R_{\max }$ ) we prescribe the normal velocity of the corresponding potential flow and zero vorticity. Time is non-dimensionalized by the factor $U_{\infty} / R$.

The equations are discretized using a vortex method Cottet \& Koumoutsakos (2000), OuldSahili et al. (2000), Cottet \& Poncet (2003), Poncet (2004), Koumoutsakos (2005), summarized as follows:

- The vorticity field is discretized on particles: $\boldsymbol{\omega}(\boldsymbol{x}, t)=\sum_{p} \boldsymbol{\omega}_{p} \delta\left(\boldsymbol{x}-\boldsymbol{x}_{p}(t)\right)$

- The particles $\boldsymbol{x}_{p}$ are convected with the flow velocity:

$$
d \boldsymbol{x}_{p} / d t=\boldsymbol{u}\left(\boldsymbol{x}_{p}, t\right), d \boldsymbol{\omega}_{p} / d t=\left[\boldsymbol{\nabla} \boldsymbol{u}\left(\boldsymbol{x}_{p}, t\right)\right]^{T} \boldsymbol{\omega}_{p}
$$

A second order Runge-Kutta scheme is used to integrate the equations of motion.

- At each time step, particles are remeshed onto a cylindrical grid. On this grid the velocity field is evaluated using a second order, FFT-based, Poisson solver and the particle strength is modified to account for vortex stretching and diffusion.

- The wall boundary conditions are translated into vorticity flux that is distributed to particles in the neighbourhood of the cylinder (see for instance Poncet (2007), Koumoutsakos et al. (1994), Cottet \& Poncet (2003)).

We note that the Lagrangian treatment of the vorticity advection method allows the use of comparatively small computational domains and large time-steps without compromising neither the stability nor the accuracy of the method in the presence of strong variations in the boundary conditions. In the optimization runs, the cylindrical $((r, \theta, z))$ computational domain was extending to $R_{\max }=1+4 \pi, L=\pi D$. For $R e=300$, the resolution was set to $256 \times 128 \times 64$ grid points. For $R e=1000$, the resolution was doubled in all directions, with a time step $\delta t=0.0625$. In the present simulations after the control velocity is turned on, the flow is computed for 100 time units with the flow settling to its steady state after 60 time units. Simulations of 100 time units require approximately 17 hours of CPU time on an Itanium 2 processor. Verification simulations were performed in a computational domain with sizes doubled in both the spanwise and radial 

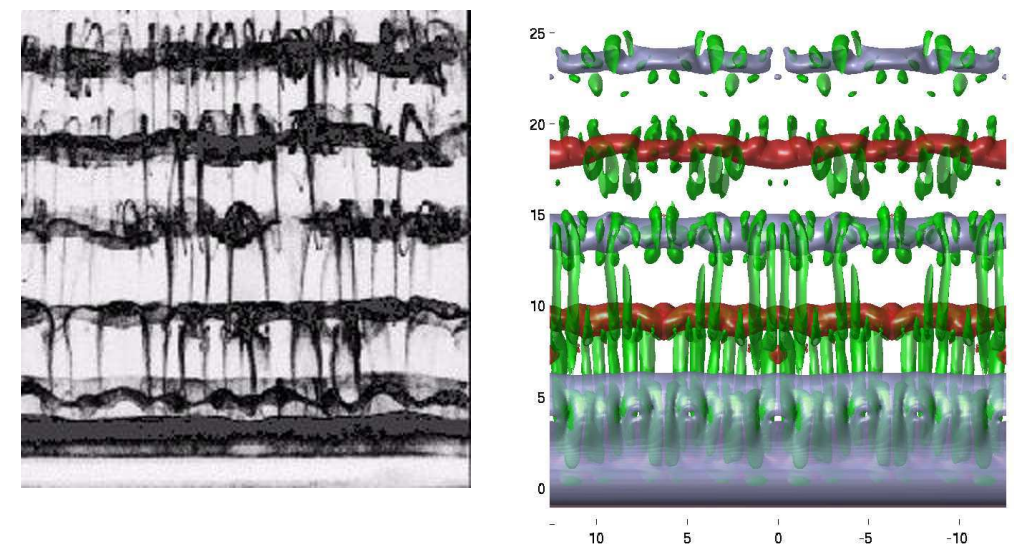

FIGURE 2. Wake representations of the flow past a circular cylinder at $R e=300$ as depicted by dye visualisations in experiments Williamson (1996b) (left), and by vorticity isosurfaces in DNS Poncet (2004) (right).

directions. These simulations were used for the flow visualization and the analysis (Figure 1) of the optimal profiles.

\subsection{Cylinder drag coefficient}

The cylinder drag coefficient is computed by taking the time derivative of the linear impulse of the vorticity field in the volume $\Omega$ surrounding the body :

$$
C_{D}=-\frac{1}{U_{\infty}^{2} R} \frac{d}{d t} \int_{\Omega} \boldsymbol{\omega} \times \boldsymbol{x} \cdot \boldsymbol{e}_{x} d v
$$

where $\boldsymbol{e}_{x}$ denotes the streamwise direction. The drag coefficient can be decomposed into contributions from the wall shear stresses $\left(C_{D F}\right)$ and the pressure gradient $\left(C_{D P}\right)$ on the surface of the body which in turn can be expressed in terms of the wall vorticity and the wall vorticity flux by :

$$
C_{D F}=-\frac{\nu}{U_{\infty}^{2} R L} \int_{\partial \Omega} \omega_{z} \sin \theta \mathrm{d} s, C_{D P}=-\frac{\nu}{U_{\infty}^{2} R L} \int_{\partial \Omega} r \frac{\partial \omega_{z}}{\partial r} \sin \theta \mathrm{d} s
$$

We note that the evolution of the drag coefficient follows closely the appearance of instabilities in the wake. These instabilities are associated with a reduced drag of the three dimensional flow. The drag coefficient is initially periodic corresponding to a 2D flow, until 3D instabilities become the dominant feature, leading to an non-periodic signal and a lower drag value (Figure 1).

The vortex method, described in the previous subsection, has been validated in Poncet (2004) in simulations of three-dimensional flows of uncontrolled cylinder wakes. The drag value of the uncontrolled flow at $R e=300$ is 1.26 . This value results form the averaging of the flow over 1200 time units. We note that this drag coefficient is within the values of 1.32, 1.24, 1.26 reported respectively in simulations Kim \& Choi (2005), Kravchenko et al. (1999), Mittal \& Balachandar (1995) and the 1.22 value reported in experiments Wieselsberger (1922) for the same Reynolds number.

\section{Drag optimization}

The present optimization strategy uses as a starting point the optimized azimuthal wall tangential velocity component, obtained in two-dimensional simulations using a genetic algorithm in Milano \& Koumoutsakos (2002). This two-dimensional profile is uniformly extended in the 

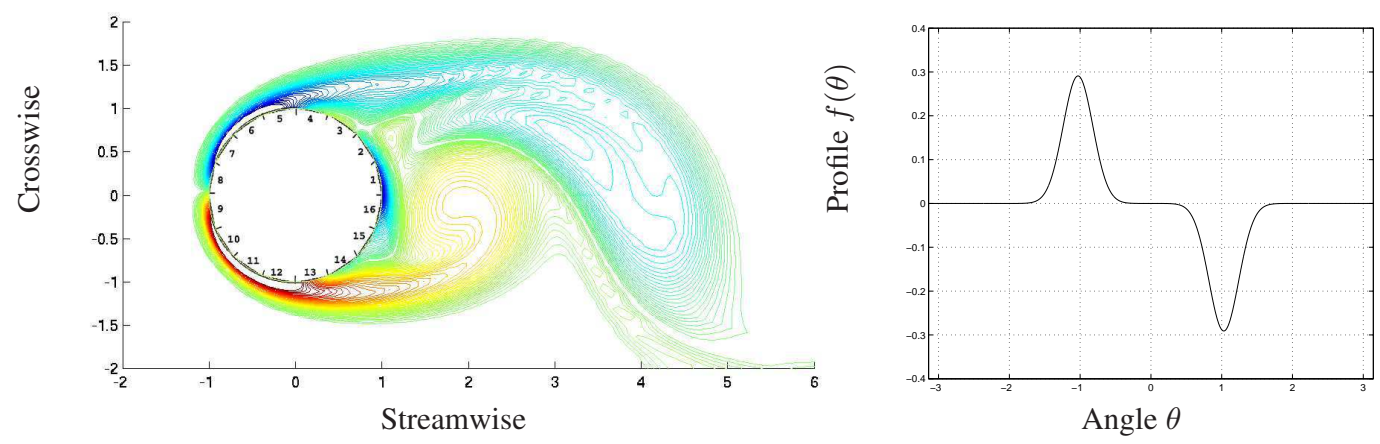

FIGURE 3. Actuator configuration on the cylinder surface (left, from Milano \& Koumoutsakos (2002)) and velocity profile fitting the best population obtained by the CGA (right).

spanwise direction of the cylinder and the optimization procedure described below identifies optimal , three-dimensional, spanwise variations for enhanced drag reduction.

\subsection{Optimization of $2 D$ cylinder wakes}

The starting point of the present optimization are the two dimensional velocity profiles obtained in Milano \& Koumoutsakos (2002) using a Clustering Genetic Algorithm (CGA). In that work the cylinder surface is divided into 16 panels, each with a steady tangential velocity that can have a value in $[-1,1]$. The CGA operates on these 16 parameters (see Milano \& Koumoutsakos (2002) for details) to identify the respective wall tangential velocities that minimize the drag coefficient. The results of this study led to a drag reduction of $50 \%$, albeit using an order of magnitude larger energy than the one reported in the present study. In addition the algorithm was able to identify in an automated fashion, that the drag reduction was largely attributed to the motion of actuators 3-4 and 13-14 (Figure 3), which contain the separation point of the uncontrolled cylinder. The velocities of the 16 actuators are in turn fitted by the following biGaussian function

$$
f(\theta)=\alpha \sum_{j=1,2} \frac{(-1)^{j}}{\sigma \sqrt{2 \pi}} \exp \left(\frac{-\left(x+(-1)^{j} m\right)^{2}}{2 \sigma^{2}}\right)
$$

with $\alpha=0.3342, m=1.03$ and $\sigma=0.21$. The arrangement of the belt actuators and the resulting fitted function are shown in Figure 3.

\subsection{Optimization of spanwise distributed controllers}

The 3D control velocity profiles of the present optimization study are spanwise modulations of the $2 \mathrm{D}$ profile given by (3.1). The velocity profile is parameterized in terms of harmonics of the cylinder span :

$$
U_{\theta}(\theta, z)=U_{\infty} f(\theta)\left(c_{0}+\sqrt{2} \sum_{k=1}^{n} a_{k} \sin (2 \pi k z / L)+b_{k} \cos (2 \pi k z / L)\right) \boldsymbol{e}_{\theta}
$$

where $L=\pi D$ is the spanwise length of the cylinder and the harmonics have a wavelength $\lambda_{k}=L / k$. We have selected $n=4$ resulting in 9 parameters for the optimization.

Our choice for the wave-number range was motivated by the findings in Poncet (2005) which considered single mode modulations of the wall tangential velocity. In that work it is shown that, at $R e=300$, three-dimensional modulations are efficient for wavelengths of up to 4 . Moreover, $k=3$ corresponds to a modulation at the closest frequency of the natural mode B instability, but it does not lead to reduced drag (except for high energy control, cf. Poncet (2005)). On the other hand, the wave $k=1$ reinforces the mode A instability, leading to a dramatic drop of the drag 


\begin{tabular}{|c|c|c|c|c|c|c|c|c|}
\hline Span & Case & \multicolumn{5}{|c|}{ Mode coefficients $c_{0}$ or $\left(a_{k}^{2}+b_{k}^{2}\right)^{1 / 2}$} & \multicolumn{2}{|c|}{$C_{D}$} \\
\hline \multirow{3}{*}{$L=\pi D$} & $I$ & 0 & 0 & 0 & 0 & 0 & 1.26 & 0.99 \\
\hline & $I I$ & 0.316 & 0 & 0 & 0 & 0 & 1.143 & 0.93 \\
\hline & $I I I$ & 0.22 & 0.139 & 0.015 & 0.011 & 0.178 & 0.862 & 0.65 \\
\hline
\end{tabular}

TABLE 1. Averaged drag : no control (Case $I$ ), 2D control (Case $I I$ ), optimal 3D control (Case $I I I)$.

coefficient. This happens despite the fact that the flow is at a Reynolds number above 250, for which mode A is no longer visible. Furthermore, this range of wavenumbers is consistent with the wavelengths obtained by shape optimization in Darekar \& Sherwin (2001), in which the most efficient perturbations are associated with modes $k>\pi / 5.6 \simeq 0.56$.

In the present optimization all parametric studies are performed at a constant energy level. The non-dimensional kinetic energy involved in the control is determined as (Poncet et al. (2005)):

$$
E_{c}^{*}=\frac{1}{4 \pi}\left(c_{0}^{2}+\sum_{k}\left|a_{k}\right|^{2}+\left|b_{k}\right|^{2}\right) \int_{-\pi}^{\pi} f(\theta)^{2} \mathrm{~d} \theta .
$$

The energy level of the present control strategy was adjusted to match the levels reported in Kim $\&$ Choi (2005). We note that the tangential profile of the blowing/suction used in this work is not available and we assume that it had an equivalent energy content as our function $f$. For a maximum blowing of 0.2 this gives an energy value of 0.1 . We thus set

$$
c_{0}^{2}+\sum_{k}\left|a_{k}\right|^{2}+\left|b_{k}\right|^{2}=0.1
$$

The present optimization algorithm proceeds as follows : For each pair of wave numbers we perform 100 function evaluations on their respective plane. The energy level $E_{c}^{*}$ is kept constant by adjusting accordingly the value of the constant mode amplitude $c_{0}$. We examine all possible pairs of wave numbers and we identify for each plane the point yielding the smallest drag coefficient. In a second stage, we use these minima as starting points for a local search based on the concept of design of experiments (Box \& Wilson (1951)). A finite difference stencil is allocated around each point in all parametric directions and a local gradient descent algorithm is performed. The algorithm is found to converge after 4 to 5 iterations.

This optimization process for the flow past a circular cylinder at $\mathrm{Re}=300$, results in a drag coefficient of $C_{D}=0.862$, corresponding to a drag reduction of about $33 \%$ (see Table 1 ). This drag reduction is four times larger than that achieved by the corresponding two-dimensional configuration. The present results compare favourably with the $23 \%$ drag reduction reported in Kim \& Choi (2005) for a maximum blowing/suction ranging between 0.1 and 0.2. In the present optimal configuration, the maximum tangential velocity was 0.09 for the case $I I$ and 0.179 for case $I I I$, with a mean velocity of 0.074 and as we optimize for a given energy level, it is not possible to match a prescribed maximum velocity. We remark that in the present case the drag reduction level scales with the energy input of the control, in contrast with the observation in Kim \& Choi (2005) that increasing the blowing/suction beyond 0.1 does not lead to further drag reduction. 

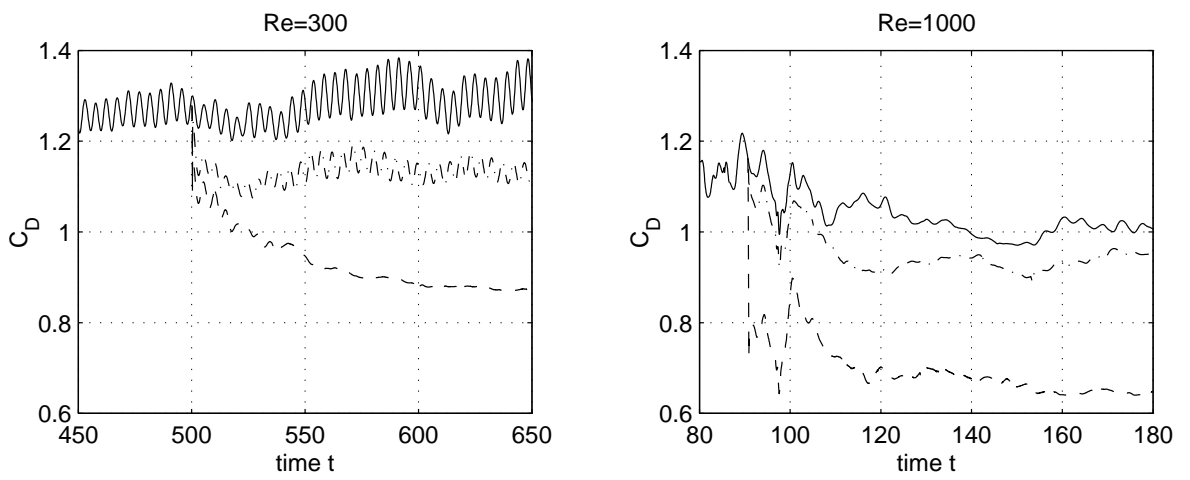

FIgURE 4. Drag time history for : uncontrolled flow (-, Case $I), 2 \mathrm{D}$ control $(-\cdot-$, Case $I I)$, and optimal 3D control (--, Case $I I I)$.
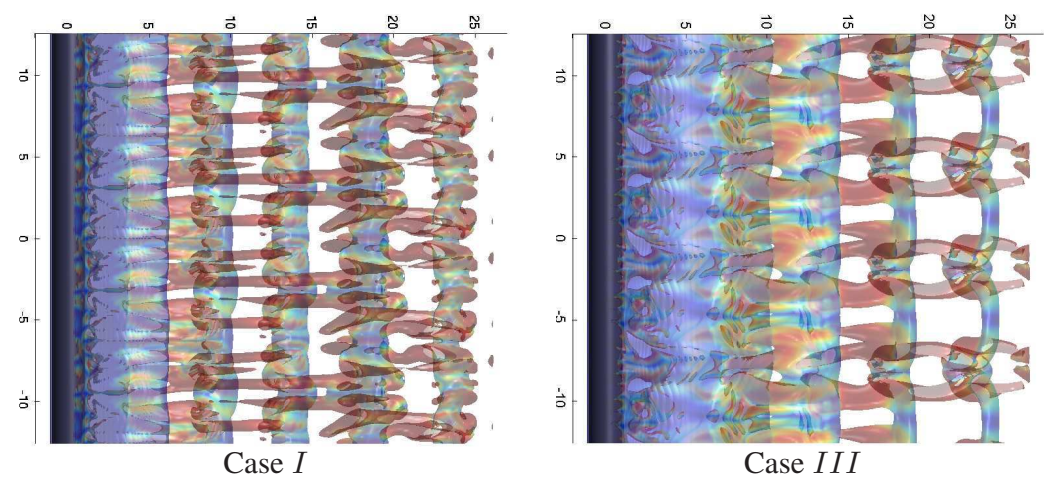

Case $I I I$
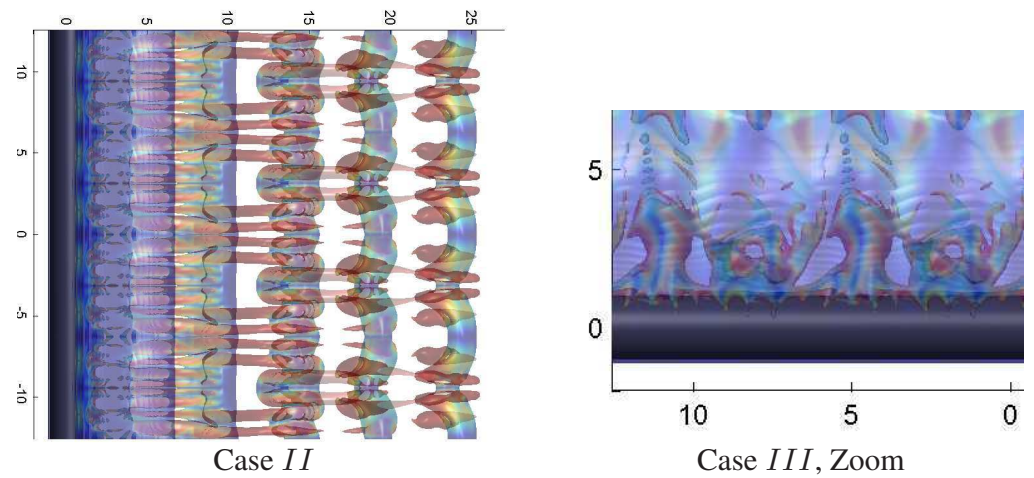

FIGURE 5 . Top views of isovorticity surfaces $\|\boldsymbol{\omega}\| R / U_{\infty}=0.3$, doubled by periodicity in spanwise direction, for the uncontrolled (Case $I$ ) (top left), 2D control (Case $I I$ ) (bottom left) and 3D control (case $I I I$ ) (right pictures) for $R e=300$.

\section{Discussion}

The results of the simulations for the uncontrolled wake (case $I$ ), the wake with $2 \mathrm{D}$ control (case $I I$ ) and the wake with optimal 3D control parameters (case $I I I$ ) are listed in Table 1. Note that for $\mathrm{Re}=1000$ we used the optimal parameters identified for $\mathrm{Re}=300$ and no further optimization was performed.

The drag reduction observed in the 3D controlled flow is analysed in terms of instantaneous vorticity isosurfaces for uncontrolled and controlled wakes (Figure 5). The effect of the 2D con- 

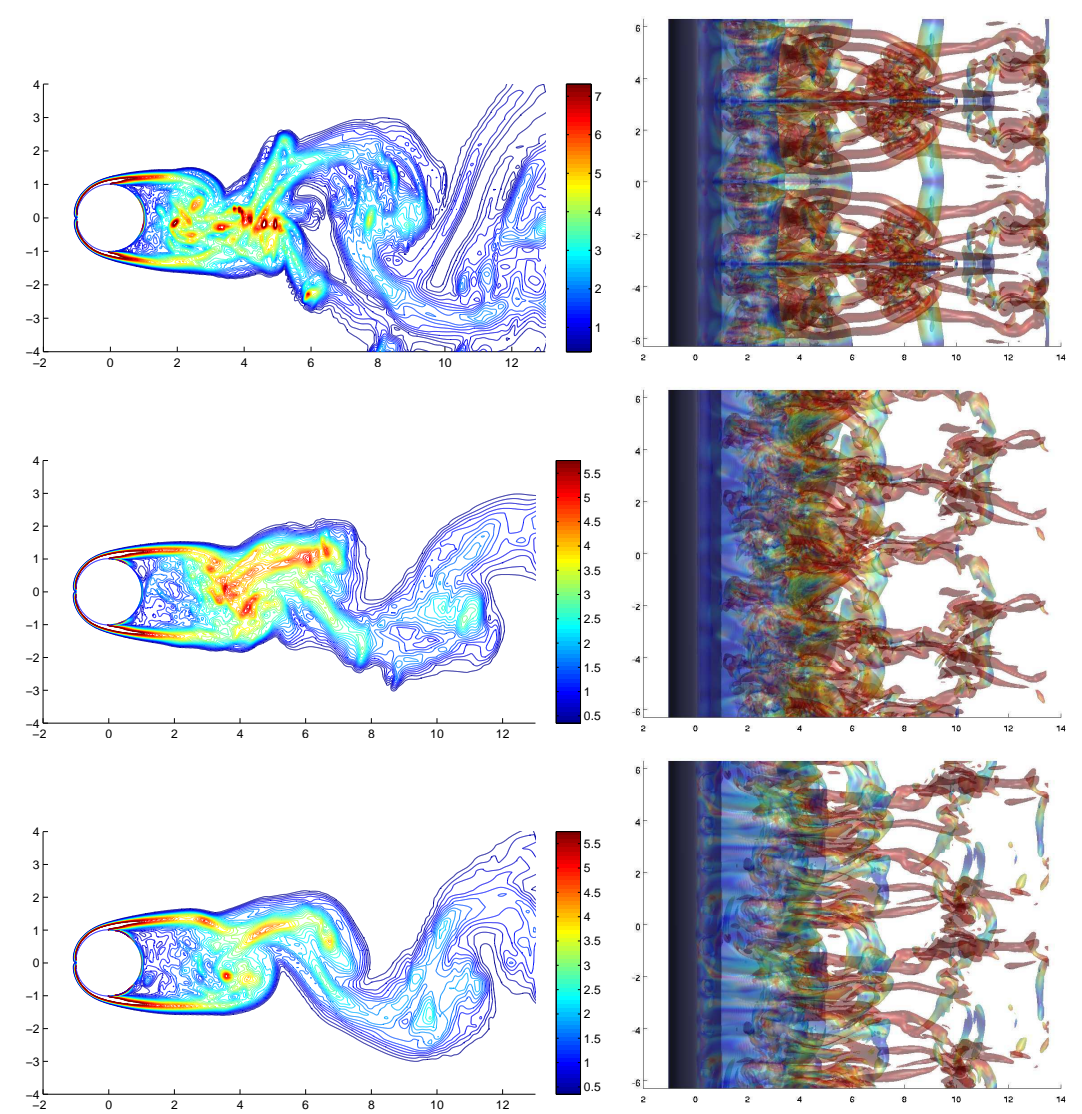

FIGURE 6. Surfaces of isovorticity $\|\boldsymbol{\omega}\| R / U_{\infty}=2.4$ (right pictures) and contours of spanwise quadratic averaged vorticity (left pictures) at $R e=1000$ : without control (top pictures), with optimal 2D control (middle pictures), and with the parameters of Case III (bottom pictures).

trol is to weaken the structures of streamwise vorticity while the optimal 3D control leads to the introduction of streamwise vorticity in the wake that further weaken the strength of the primary spanwise vortices. Furthermore, in the 3D control, the von Kármán alleys are attached longer to the cylinder surface thus delaying their shedding in the wake. This delayed shedding is visible in the absence of oscillations in the drag evolution for case $I I I$ on Figure 4. Zooming on the vorticity isosurface for case $I I I$ (Figure 5) we observe that strong braids of streamwise vorticity act to attach the primary vortices to the surface of the cylinder. The normal vorticity on the cylinder is correlated with spanwise variations of the slip velocity on short wavelengths. This result further justifies the choice for harmonic, rather than subharmonic control parameters, that further enhance the natural cylinder wake instabilities.

We explored the validity of this mechanism for higher Reynolds number flows by performing a DNS at $R e=1000$. We observe that the control parameters, identified for $\mathrm{Re}=300$, lead also to drag reduction for this higher Reynolds number. This drag reduction is much more significant than that obtained by 2D control (see Figure 4). On Figure 6 we present contours of cross section for the vorticity magnitude averaged in the spanwise direction and the instantaneous vorticity isosurfaces. Both 2D and 3D control clearly result in a strong reduction of the strength of the vortices in the wake. The 3D control, although it tends to reinforce 3D instabilities, results in a smoother organization of the near wake, with strong streamwise vortex braids that are attached to the body. An inspection of the phase diagram of the friction and pressure drag (Figure 7), 

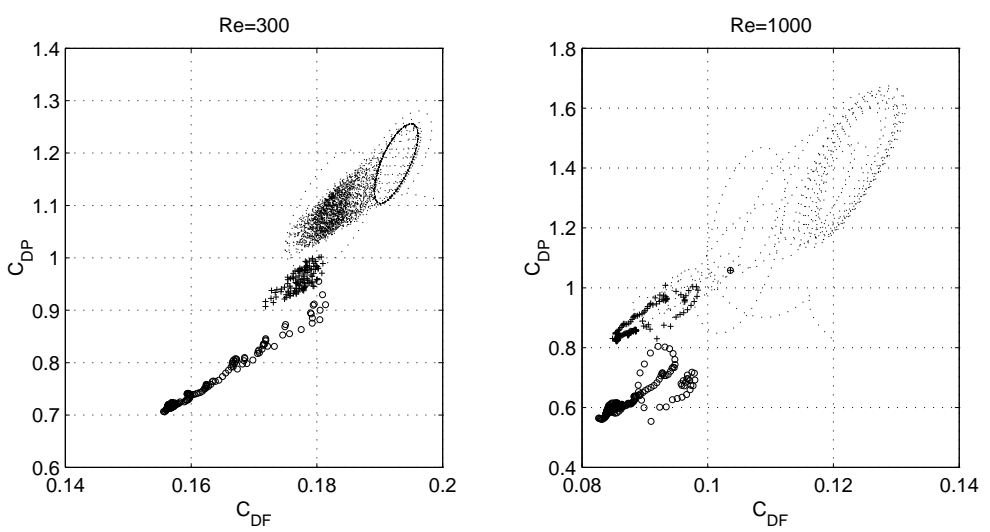

FIGURE 7. Drag coefficient pressure part $C_{D P}$ with respect to friction part $C_{D F}$ for $R e=300$ (left picture) and $R e=1000$ (right picture), for uncontrolled flow (- -), two-dimensional control (+) and three-dimensional control (o).

shows that for $R e=300,2 \mathrm{D}$ and 3D control lead to a systematic drop of pressure drag and to an asymptotic state for which both friction and pressure are decreased. For $R e=1000$, the 2D control does not change significantly these quantities, while the 3D control acts mainly on the pressure drag.

As shown in Table 1 the optimal configuration, modes 1 and 4 have coefficients that are an order of magnitude larger than those for modes 2 and 3. Since these modes are close to natural instability modes A and B (respectively of wavelength $\lambda_{1} / D \simeq 4$ and $\lambda_{4} / D \simeq 1$ ) and as mode A is associated to a natural drag reduction at low Reynolds numbers, we performed complementary simulations at $R e=300$ with a cylinder span of $L=4 D$, thus matching the wavelengths of modes A and B. Using the coefficients of Run $I$, which are probably not optimal for this span, we obtained a drag coefficient of 0.90 . We next performed a simulation keeping the same coefficient values for $k=0, k=1$ and $k=4$ and turning off modes 2 and 3 . We obtained a similar drag value of 0.88 . These results confirm the importance of exciting modes A and B for drag reduction. The present optimization process identified in an automated fashion this physical property of the system and allocated the proper energy distribution between these modes and the 2D optimal profile to achieve maximal drag reduction.

\section{Conclusions}

We have demonstrated that, for a prescribed energy level, appropriate spanwise distribution of tangential velocities can lead to significant drag reduction. The optimal control parameters have been systematically identified for a low Reynolds number flow, and they have been shown to be also effective at moderate Reynolds numbers. The driving mechanism is the generation of strong streamwise vortex braids that delay the shedding and weaken the spanwise vorticity in the wake. The resulting optimal parameters indicate that the three dimensional disturbances, corresponding to the modes A and B of three dimensional wake instabilities, are largely responsible for this drag reduction.

\section{Acknowledgments}

We wish to acknowledge many discussions with Prof. Michele Milano (Arizona State University) as well as several helpful suggestions from the reviewers. The second author was supported 
by the French Ministry of Education through ACI grant MD-412002 which also partially provided the computational resources used in this project.

\section{REFERENCES}

BARKLEY, D. \& HENDERSON, R. D. 1996 Three-dimensional Floquet stability analysis of the wake of a circular cylinder. J. Fluid Mech. 322, 215-241.

Box, G. E. P. \& WILSON, K. B. 1951 On the experimental attainment of optimum conditions. J. of the Royal Stat. Soc. 13:B, 1-38.

Choi, H., Jeon, W.-P. \& Kim J. 2008 Control of flow over a bluff body. Ann. Rev. Fluid Mech. 40.

Cottet, G.-H. \& Koumoutsakos, P. D. 2000 Vortex methods, theory and practice. Cambridge University Press.

Cottet, G-H. \& Poncet, P. 2003 Advances in Direct Numerical Simulations of three-dimensional wallbounded flows by Vortex In Cell methods. J. Comp. Phys 193, 136-158.

Cottet, G-H. \& Poncet, P. 2004 New results in the simulation and control of three-dimensional cylinder wakes. Comp. Fluids 33, 697-713.

DAREKAR, R. M. \& SHERWIN, S. J. 2001 Flow past a square-section cylinder with a wavy stagnation face. J. Fluid Mech. 426, 263-295.

Dennis, S. C. R., Nguyen, P. \& KocABiyik, S. 2000 The flow induced by a rotationally oscillating and translating circular cylinder. J. Fluid Mech. 385, 255-286.

Dobre, A., HANGAN, H. \& ViCKerY, B. J. 2006 Wake control based on spanwise sinusoidal perturbations. AIAA J. 44(3), 485-492.

KIM, J. \& CHOI, H. 2005 Distributed forcing of flow over a circular cylinder. Phys. Fluids 17(3), 033103.

Koumoutsakos, P. 2005 Multiscale flow simulations using particles. Ann. Rev. Fluid Mech. 37, 457-487.

Koumoutsakos, P. , LeOnARD, A. \& PePin, F. 1994 Boundary conditions for viscous vortex methods. J. Comp. Phys. 113, 52.

Kravchenko, A. G., Moin, P. \& Shariff, K. 1999 B-spline method and zonal grids for simulations of complex turbulent flows. J. Comput. Phys. 151, 757-789.

LEE, S. J., LIM, H. C., HAN M. \& LEE, S. S. 2005 Flow control of circular cylinder with a V-grooved micro-riblet film. Fluid Dyn. Res. 37(4), 246-266.

LIM, H. C. \& LEE, S. J. 2004 Flow control of a circular cylinder with O-rings. Fluid Dyn. Res. 35(2), $107-122$.

LUO, S. C. \& XIA, H. M. 2005 Parallel vortex shedding at Re = O (10(4)) - a transverse control cylinder technique approach. J. Fluid Mech. 541, 134-165.

Milano, M. \& Koumoutsakos, P. 2002 A clustering genetic algorithm for cylinder drag optimization. J. Comp. Phys. 175, 79-107.

MitTAL, R. \& BALACHANDAR, S. 1995 Effect of three-dimensionality on the lift and drag of nominally two-dimensional cylinders. Phys. Fluids 7, 1841.

Ould-SAhili, M. L., COTTET, G.-H. \& El Hamraoui, M. 2000 Blending finite-differencies and vortex methods for incompressible flow computations. SIAM J. Sci. Comp. 22, 1655-1674.

PONCET, P. 2004 Topological aspects of the three-dimensional wake behind rotary oscillating circular cylinder. J. Fluid Mech. 517, 27-53.

Poncet, P., Cottet, G-H. \& Koumoutsakos, P. 2005 Control of three-dimensional wakes using evolution strategies. C. R. Mecanique 333, 65-77.

Poncet, P. \& KoumoutsaKos, P. 2005 Optimization of vortex shedding in 3D wakes using belt actuators. Intl. J. Offshore Polar Eng. 15 (1), 7-14.

PoncET, P. 2005 Bimodal optimization of three-dimensional wakes. Proceedings of the 6th DLES Conference, Springer-Verlag, Poitiers, France, 449-458.

PONCET, P. 2007 Analysis of direct three-dimensional parabolic panel methods. SIAM J. Numer. Anal. 45, 2259-2297.

Tokumaru, P. \& Dimotakis, P. 1991 Rotary ooscillation of a cylinder wake”, J. Fluid Mech. 224 , 77-90.

WIESElSBERger, C. 1922 New Data on the Laws of Fluid Resistance. Report NACA-TN-84.

Williamson, C. H. K. 1996 Vortex dynamics in the cylinder wake, Annual Review of Fluid Mechanics 28, 477-526. 